\section{Limitation du rôle de l'Energie électrique dans la métallurgie du fer}

Nous extrayons d'une très intéressante conférence fatto tout dernièrement à la Société Industrulle de l'Est, par M. Gustave Gin, l'électrométallurgiste bien connu, les considérations suvantes qui sont de nature à particulièrement intéresser nos lecteurs.

L'électricité doit être généralement considérée comme une énergie de luxe, dont il faut restreindre l'emploi aux seuls opératıons pour lesquelles elle se montre netiement supérieure ou non remplaçable, et l'on ne peut la prodiguer que dans les régions privlégiées où les grandes chutes permetlent de la produire à bas prix, sans qu'elle puisse trouver sur place des utilisations plus avantageuses.

Il est du reste loisible de limiter le role que l'on entend réserver à l'énergie éleclrique, car il est aussi possible de borner son intervention à la mise au point d'un acier presque élaboré dans les appareils connus, que de réalıser la fabricalion intégrale en partant du minerai pour aboutir à un acier de composition quelconque.

Entre ces deux limites, le four électrique peut parcourir toute Ja gamme des opéralions productrices de l'acier et seules les considérations économiques peuvent étendre ou restreindre son champ d'action.

Les inventeurs, qu'attire la difficulté, se sont attaqués d'abord à la fabrication intégrule, et comme le problème était aussi ardu que complexe, la solution s'est fait allendre. Plus tard, les expériences ont porté sur le seul affinage de la fonte brute, prise à l'état solıde ou préalablement fondue; puis on a essayé la rabrication au moyen des riblons, qui est plutôt une fusıon qu'un affinage.

Enfin, on est arrivé à éliminer non seulement la réduction du mineral et la fusion du métal, mais encore l'affinage préalable, pour ne laisser à l'électricité qu'une sorte de suraffinage ou de mise au point finale.

On voit combien élastique est le rôle du four électrique, qui peut opérer seul la fabrication de l'acier, ou la réaliser en association avec le haut-fourneau, ou bien encore collaborer à celte fabricalion à la suile du haut-fourneau et du convertisseur.

Des expériences faltes jusqu'à ce jour, il résulte qu'à ces diverses fabrications correspondent les dépenses sulvantes d'énergie:

Fabrication intégrale en partant du minerai.

Fabrication au moyen de la fonle solide.....................

Fabrication par la fonte et les riblons solides.

Fabrication par la fonte liquide....

Fabrication après convertissage

sommare...................

\section{$2.800 \mathrm{a} 3.200 \mathrm{KW} . \mathrm{H}$}

\subsection{0 à $1.100 \mathrm{KW} . \mathrm{H}$}

850 à $1.100 \mathrm{KW}$. H

450 à $600 \mathrm{KW} . \mathrm{H}$

\section{$200 \mathrm{a} \quad 300 \mathrm{KW} . \mathrm{H}$}

Et me voici, Messieurs, lout naturellement appelé à vous parler de ce groupement idéal, du haut-fourneau réducteur avec le four électrique épurateur, qui utiliserait comme source de force motrice les chaleurs perdues des gaz des hauts-fourneaux.

En théorie, il devrait suffire de 300 kilogs de combuslible pour extraire et fondre une tonne de fer par réduclion de l'oxyde $\mathrm{Fe}^{2} \mathrm{O}^{3}$. En 1850, avec les hauts-fourneaux de 20 tonnes, on consommait encore 3 tonnes de coke par tonne de fonte coulée. En 1863, avec les premiers hauts-fourneaux de 60 tonnes, on descendit à $1500 \mathrm{kgs}$. Actuellement, la consommalion moyenne est voisine de $1000 \mathrm{kgs}$ et tend à descendre vers $800 \mathrm{kgs}$.

Cette économie dans la consommation du combustible est dûe à l'augmentation de la capacité des hauts-fourneaux et de la pression du vent et surtout au chauffage du vent par les récupérateurs ulilisant la combustion des gaz mêmes du haut-fourneau.

Mais, quelle que solt la qualité du coke brúlé par tonne de métal élaboré, Ja plus grande partie de l'énergie disponible dans le combustible en excès serait encore dissipée en pure perte dans les gaz qui s'échappent des hauls-fourneaux, sı l'on n'avalt eu l'idée de les utilıser, d'abord pour la produchon de la vapeur el ensuite pour la combustion directe dans les moteurs à explosion.

Cette dernière application n'a pas été facılement résolue, car elle devait vauncre de nombreux obstacles résultant, d'une part, du faible pouvoir calorifique des gaz et de la difficulté d'allumage qui en résulte, et d'autre part, de la teneur de ces gaz en vapenn d'eau et en poussières minérales ou métalliques entrainées.

Les difficultés d'allumage ont élé surmontées par une compres. sion plus élevée et les poussières supprimées par une épuration mécanique poussée aussi loin que possible. Quant à l'ear entrainée, on s'en débarrasse maintenant par les appareils congé. lateurs de Gayley, qui fonctionnent aux Etats-Unis.

Bref, on est arrivé maintenant à construire des moteurs utili. sant le gar des hauts-fonrneaux el développant une puissance de 1500 et même $2000 \mathrm{HP}$.

La récupération d'énergie sur laquelle on compte actuellemeni est telle qu'un haut-fourneau, produisant journellement 100 tonnes de coke, pourrait alimenter simultanément une puissance motrice de 2500 chevaux-vapeur, dont 350 seraient consacrés à la machine soufflante et 150 aux appareils d'épuration du gaz, tandis que $2000 \mathrm{HP}$ resteraient disponbles pour d'autres utilisations, ef notamment pour la production d'énergie électrique.

Si l'on songe que la production mondiale de la fonte atteinl actuellement $\mathbf{4 0}$ millions de tonnes, on en déduil qu'elle pourrai! fournir comme sous-produit une quantilé d'énergie équivalante au travail permanenl de plus de 2 millions de chevaux-vapeur.

Au surplus, il faut observer que cette puissance supplémentaire obtenue avec les moteurs à explosion ne se traduit pas entière. ment en bénéfice. Elle exige, pour les appareils d'épuration, les pompes, les moteurs et les bâtiments qui les abrilent, une immo. brlisation qui ressort à plus de $300 \mathrm{fr}$. par cheval. Mais, en tenant compte de cette immobilisalion qu'il faut amortir, et en ajoutant à l'amortissement les frais d'entretien, de surveillance et de grais. sage, on arrive, en somme, pour le cheval-heure, à un prix de revient qui n'est peut-être pas supérieur à un centime. Dans dess conditions, l'utilisation de l'énergie supplémentaire devient pos. sible pour la fabrication de l'acier électrique et elle peut, dans̆ une cerlane mesure, lulter contre l'énergie oblenue à très bas prix pour l'aménagement des hautes chutes d'eau.

Si l'on se reporte aux chiffres que j'ai donnés pour les diverses. interventions possibles de l'électricité dans la fabrication de l'acier, on voit que les dépenses correspondantes d'énergie élec. trique par tonne fabriquée s'établissent comme suit :

Fabrication en partant de la fonte liquide sortant du haut-fourneau . ............................ Fabricalıon après affinage préparatoire au convertisseur

ou au Martın ............................. 2 à 3 fr.

Il ne semble pas que la question de dépense d'énergie électrique puisse effrayer aucur industriel, si, comme il est maintenan! certain, la fabrication ou la mise au point électrique de l'aciet? doit condurre à l'obtention d'un métal de qualité supérieure ét plus régulière.

En supposant que la puissance totale correspondanle aur chaleurs perdues des hauts-fourneaux soit ulılisée pour la fabrication de l'acier en partant de la fonte liquide, et en admetian une dépense maxima de $550 \mathrm{KW}$. H. par tonne, on pourrait pro. duire annuellement près de 20 millions de lonnes d'acier, c'est-d* drre transformer en acier la moitié de la production mondiale de fonte. Si l'on procédalt à un affinage sommaire au convertisseul suivi d'une épuration complémentaire dans le four électrique solution que je proposais déjà en 1902, il se pourrait que la lot. lité de la fonte puisse être transformée en acier, le haut-fournên réalisant cet ensemble, admirable de conditions, d'élaborel métal fondu et de fournir d'autre part toute l'énergie nécessaim pour sa transformation en acier.

Il est parfaitement inutile d'espérer que des transformations aussi radicales se produisent jamais, car les progrès de l'industri ne s'accomplissent pas avec un ensemble et un accord auss parfaits, et, d'autre part, ce qui est un progrès aujourd'hui pell ne plus l'être demain. Aussi n'ai-je formulé les hypothèses qut vous venez d'entendre que pour exprimer, sous une forme sub gestive, toul l'intérêt qui me paraîl s'allacher à cetlè donble question de l'utilisation des chaleurs perdues des hauts-fourneal el de son application à la fabrication de l'acier électrique.

Et, pour conclure, maintenant, je veux, avec une légitimi fierté, faire ressortir la place prépondérante que les Français onl su prendre dans les progrès de l'électrométallurgie du fer. Mlạis je ne parle que des électrométallurgistes, car la métallurgie proprement dite n'a pas encore pris nettement position. Elle obsente et elle altend, risquant certainement de se laisser devancer part de plus hardis el de plus entreprenants.

on m'a dit plusieurs fois en Allemagne : 
"On ne peut nier que les Français soient les initıateurs de l'électrométallurgie du fer, mais ils ne l'appliqueront qu'après les Allemands et les Américains ".

on nous accorde l'esprit d'intuition et de synthèse, la conception claire el limpide qui fait les inventeurs, mais on nous refuse l'esprit pratique, la patience et la persévérance exigées pour l'application industrielle. On dıt aussi que nous n'avons pas confiance en nous-mêmes. Mais, que ne diton pas?

Quant à moi, je ne crois pas, je ne veux pas crorre, que nous soyons en voie de décadence industrielle, et il suffirart de l'admirable essor de l'industrie automobile, crée et continuée par nous, pour prouver que nous sommes loujours à l'avant-garde du progrès et que nous savons nous y maintenır.

Il en sera de mẻme pour ce qui concerne l'acier électrique; je le crois et je le dis : j'ai la ferme espérance que ce sera encore le coq gaulois qui chantera au sommel de l'édifice elevé prerre à pierre par les Français et achevé par eux.

G. GIN.

\section{Quelques Projets Gigantesques}

La presse technique en France et à l'étranger a récemment publié les informations suivantes qu'à notre tour nous ne pouvons manquer de reproduire sous peine de paraître ignorant des grands projets.

Il s'agir d'abord d'un projet dû à $M$. Guarini, professeur à l'Ecole des Arts et Métiers de Lima, qui propose l'utilisation industrielle des eaux du lac Titicaca. S'étendant sur une superficie de 6630 kilomètres carrés, sous une profondeur moyenne de 20 mètres, ce lac constitue un réservoir de 132600000 mètres cubes. L'auteur de ce gigantesque projet indique la possibilité de canaliser les eaux de ce réservoir naturel vers la côte du Pacifique, assez proche, en suivant les pentes rapides de la Cordillière des Andes. Le lac étant à 3800 mètres d'altitude, on pourrait, en dérivant 100 mètres cubes par seconde, produire au moins 2 millions de chevaux pratiquement (?) utilisables sur l'arbre des turbines.

Les moyens proposés par M. Guarini pour opérer cette dérivation sont, en principe, très simples. Le lac étant à 250 mètres en dessous du point le plus bas de la chaîne de montagnes quile sépare de la côte, on a le choix, dit-il, entre un tunnel perçant la montagne en ligne droite et le dispositif suivant qui serait plus élégant, et peut-être moins coûteux : des pompes actionnées par l'énergie des chutes qui seraient établies à la suite les unes des autres, entre le sommet de la montagne et la côte, relèveraient l'eau du lac à 250 mètres et, de là, cette eau s'écoulerait vers le Pacifique en traversant une série d'usines échelonnées sur une hauteur totale de plus de 3000 mètres; au rendement près des pompes et des conduites, on récupérerait ainsi l'énergie dépensée à l'élévation. Après a voir traversé les dernières turbines, l'eau servirait à l'irrigation des cuitures de la côte.

D'après un avant-projet sommaire, le coût des installations serait d'un ordre de grandeur qui ne dépasse pas l'inagination, puisqu'avec 200 millions, paraît-il, on pourrait peut-être en voir le bout! Cela mettrait les frais de premier établissement du cheval à 100 francs. Nous connaissons plus d'un auteur de projets qui, dans nos Alpes, a fait ressortir les frais d'installation du cheval a moins de 100 francs sur l'arbre des turbines. Ce prix n'est donc pas exagéré dans le sens du bon marché qu'on pourrait s'attendre à voir ressortir de ces grandioses conceptions auxquelles nos confrères du Nouveau-Monde nous ont quelque peu habitués. Mais ce que nous ne chercherons même pas à mettre en comparaison avec ce que nous connaissons de nos pauvres petites installations alpestres, ce sont les éléments d'appréciation des puissances aménageables et les moyens de construction. En Amérique, comme on le sait, tout est grand, et nous aurions mauvaise grâce, nous petits alpins, à discuter des projets de géants.

Cette énorme quantilé d'énergie pourrait être utilisée à la traction électrique des chemins de fer, à l'électrochimie et à l'électrométallurgie, aux services des mines du Pérou et de l'agriculture, rotamment au labourage électrique, à l'éclairayge des localités et enfin à la fabrication des nitrates pour concurrencer les gisements naturels du Chili.

Que le Niagara se tienne bien... ou il est enfoncé !

Mais il n'y a pas que les Américains pour faire grandement les choses; nous aussi, en Europe, sans vouloir leur disputer le record des chutes monstres, nous pouvons néanmoins leur opposer des forces hydrauliques respectables.

Le projet suivant, dû à $M$. Von Dowat, permettrait de créer, pas bien loin d'ici, 300000 chevaux, tout simplement. Cette fois, il nous est facile d'y aller yoir, c'est en Bavière, Et nous qui mettions l'Allemagne au rang des pays peu fortunés sous le rapport de la houille blanche !...

Voici, dans ses grandes lignes, l'économie du projet"en ques. tion: sur l'Isar, à moitié chemin entre Wallgan et Vorderiss, on construirait un barrage - de dimensions telles que jamais circulaire ministérielle n'en a encore prévu chez nous - et qui retoulerait les eaux de la rivière jusque dans un petit lac alpestre de 650 miètres de large et 6 kilomètres de long. Ce serait là un premier réservoir emmagasinant 65 millions de mètres cubes. De ce lac, l'eau serait dirigée au moyen d'un tunnel de 3800 mètres, dans le lac Walchen, situé à 52 mètres plus bas. La réalisation de cette différence de niveau dans une chute hydraulique donnerait dabord 20000 chevaux. Par une seconde galerie, les eaux du Walchen seraient envoyées dans le lac Kochel en franchissant une seconde chute de 203 mètres où l'on recueillerait 80000 chevaux.

Ce lac reçoit aussi les eaux du Loisach, torrent à débit très variable. On établirait alors un barrage sur ce torrent, pour créer un lac artificiel de Tscheulohe à Oberau et capable de contenir 100 millions de mètres cubes. Ce réservoir pourrait fournir un débit de 63 mètres cubes à la seconde qui, joints aux 23 mètres cubes fournis par l'Isar et ses affluents, donnerait un débit total de 86 mètres cubes par seconde. Ce volume pourrait être utilisé sous une nouvelle différence de niveau de 300 mètres permettant d'obtenir 200000 chevaux dans cette troisième chute.

Au total, on aurait ainsi 300000 chevaux disponibles.

Cela ne vaut pas le Titicaca, mais enfin c'est déjà une puissance capable de satisfaire à quelques-unes des demandes d'énergie que la paisible industrie de notre vieille Europe réclame pour se maintenir au niveau de celle du Nouveau Monde.

Il paraît que les Bavarois discutent ferme, non pas tant sur la possibilité de mener à bien cette entreprise, du reste parfaitement exécutable sous le rapport technique, que sur le point de savoir si ces barrages, ces usines avec leurs tuyaux monstres et leurs réseaux de fils et de poteaux, ne nuiront point aux charmes du paysage, ne "matérialiseront "pas trop cette idéale région des Alpes, célèbre par le pittoresque de ses sites, aussi bien que par les fêtes artistiques auxquels ils servent de cadre? On se demande si les chutards et les électriciens n'éloigneront pas a jamais les poètes et les musiciens de Bayreuth !...

Il s'agit ensuite du transport électrique de l'énergie des chutes du Zambèze, aux Victoria Falls, jusqu'aux mines du Rand, dans le Transval: la distance est de 1200 kilomètres!

D'après certaines estimations, la puissance des chutes du Zambèze aux Victoria Falls serait d'environ 500 ooo chevaux, pour une chute utile de 100 mètres. L'Afrique, on le voit, rivalise avec l'Amérique. Comme d'une part cette puissance ne parâ̂t pas immédiatement utilisable sur place - et pour cause - que d'autre part le charbon coûte très cher au Transvaal, M. Fox, directeur de la British South African Company, a étudié le projet peu banal de transporter une partie de cette énergie jusqu'aux mines du Rand qui absorbent actuellement I 50 ooo chevaux, paraît-il.

M. Fox adopte le système Thury à courant continu-série avec une tension de roo ooo volts qu'il estime ne pas devoir présenter plus de difficultés d'isolement que le courant triphasé 